\title{
Management and outcome of infective prepatellar bursitis
}

\author{
James Wilson-MacDonald \\ Accident Service, John Radcliffe Hospital, Oxford, OX3 9DU, UK.
}

\begin{abstract}
Summary: Forty seven cases of prepatellar bursitis are reported. Twenty one patients had sustained a recent injury with a break in the skin which had caused the infection and seventeen patients were employed in jobs which involved kneeling. Oral antibiotics proved to be inadequate treatment in many cases. Splintage and intravenous antibiotics with or without aspiration of the bursa were usually successful in treating the condition, although nine patients required surgical drainage of the bursa. Twelve patients continued to have symptoms months or years after the infection, particularly those with preexisting chronic bursitis, or those who kneeled at work. There was little difference in the results between the different treatment groups.
\end{abstract}

\section{Introduction}

Chronic prepatellar bursitis is a relatively common condition and the results of treatment are well documented. However only twenty cases of infective prepatellar bursitis are reported in the literature, excluding cases of beat knee seen in miners. ${ }^{1-5}$ The management and results of treatment in 47 cases are reported.

\section{Patients and methods}

All patients presenting to the Accident Service of the John Radcliffe Hospital, Oxford with a diagnosis of infective prepatellar bursitis between April 1984 and March 1986 were included in the study. Those presenting in the first year (13 patients) were studied retrospectively whereas those presenting in the second year (34 patients) were studied prospectively. All the patients were sent a postal questionnaire some months after their discharge from hospital, enquiring about the duration of pain or other symptoms after discharge from hospital, time off work and any precautions taken to prevent a recurrence of the infection.

\section{Results}

Forty one patients were men and the average age was 36 (range 16-72). Seventeen patients were employed in work which involved kneeling such as carpet laying, or painting and decorating, and of these five had had a

Correspondence: J. Wilson-MacDonald M.B., Ch,B., F.R.C.S.

Accepted: 23 April 1987 recent penetrating injury to their knee whilst at work. Five patients had a history of chronic prepatellar bursitis and all of these had jobs which involved kneeling.

All patients presented with a painful prepatellar swelling and all patients had either a fever or a cellulitis around the prepatellar bursa. The average duration of symptoms at presentation was two days (range 1-14 days). Two patients had a sympathetic effusion of the knee joint, of whom one underwent aspiration of the joint to exclude a septic arthritis.

Forty two patients were admitted immediately from the Accident Department of whom 14 had seen their general practitioners in the previous week and had been treated with antibiotics. Five patients were initially treated as outpatients in the Accident Service, but three of these subsequently required admission for further treatment. Thus 19 patients had been treated as outpatients either by their general practitioner or by the Accident Service.

All patients admitted to hospital were treated with intravenous antibiotics, splintage of the knee and elevation. Twenty seven patients underwent aspiration of the bursa, and of these three required surgical drainage due to failure of treatment. Six patients were treated with surgical drainage as the primary treatment, and 14 patients had no drainage of the bursa. All the surgical wounds were healed within 3 weeks of incision.

Twenty two of the thirty three bursae aspirated or drained surgically grew bacteria on culture, of which sixteen grew Staphylococcus aureus, five grew Streptococcus pyogenes and one grew Staphlococcus epidermidis. Of eleven bursal aspirations which grew no bacteria on culture, nine were in patients who had been 
given antibiotics by their general practitioner during the preceeding week.

Forty of the patients were contacted. The average time taken off work was 18 days, and the majority of patients had symptoms for less than 5 weeks. Twelve patients were still symptomatic at follow-up with aching, swelling, pain or stiffness. Eight of these patients were employed in kneeling jobs, and all of the patients with a history of chronic prepatellar bursitis were symptomatic at follow-up. The method of treatment did not seem to influence the long term results. None of the patients had changed their employment as a result of the infection, but three symptomatic patients were using knee pads at the time of follow-up.

\section{Discussion}

Prepatellar bursitis is a relatively common disease with an incidence of more than 20 per year in the Oxford region (population 460,000 ). The condition called beat knee (chronic prepatellar bursitis) is very common in miners. ${ }^{6,7}$ Poor hygiene and skin infections can cause infection in miners with beat knee. ${ }^{8}$. Penetrating injury is a rare cause of infection in beat knee, ${ }^{6-8}$ whereas $43 \%$ of the patients in this study and between $58 \%$ and $68 \%$ of those reported by other authors had had a recent penetrating injury in the prepatellar region. ${ }^{2-4}$ Chronic prepatellar bursitis was present in five of the patients reported here, none of whom had had a penetrating injury. The condition of infective prepatellar bursitis seen in these patients would seem to correspond to the condition of beat knee, and should probably be considered an industrial disease.

Outpatient treatment of infective prepatellar bursitis is probably contraindicated and early and energetic treatment is necessary to adequately treat the disease. Seventeen of the nineteen patients initially treated as outpatients (either by their general practitioner or by the Accident Service) subsequently required admission for splintage, intravenous antibiotics and elevation. Antistaphylococcal and antistreptococcal antibiotics should be used, and the bursa should be aspirated where there is palpable fluid. Repeated aspiration often controls the infection where there is recurrence of fluid in the bursa. ${ }^{1-5,7-9}$ Where aspiration fails or where there is already a pointing abscess, incision and drainage of the bursa is indicated. Two of the patients had a sympathetic effusion in the knee joint. Septic arthritis of the knee should be easy to differentiate from prepatellar bur-

\section{References}

1. Canosso, J.J. \& Sheckman, P.R. Septic subcutaneous bursitis: report of sixteen cases. $J$ Rheumatol 1979, 6: 296-302. sitis, ${ }^{3}$ and aspiration of the joint should be discouraged unless there is a strong suspicion of septic arthritis thus avoiding the possible introduction of organisms. into the joint.

Surgical treatment of the prepatellar bursitis has poor reputation. ${ }^{10}$ Morbidity after surgery is usually related to ill placed incisions and generally a laterấf parapatellar incision is recommended. . $7,70,11 \mathrm{Nin}$ patients underwent incision and drainage of the bursa Of these, eight underwent incision directly over the bursa and one underwent incision through a lateraf parapatellar incision. Although three of these patientse were still symptomatic at follow-up, all three had has previous prepatellar bursitis and were in employme where they needed to kneel. Incisions directly over the patella in septic bursitis seem to cause few symptomis once the wound is healed, but where there is no abscess pointing anteriorly over the patella a laterat parapatellar incision is probably best.

Previous authors claim no symptoms at follow-up in their patients. ${ }^{2-4}$ This study would suggest that long standing symptoms are fairly common after infection in the prepatellar bursa. The majority of patients whఖ were symptomatic at follow-up had jobs whic involved kneeling, most of whom had had chronis bursitis in the past.

Four patients were using knee pads at work at time of follow-up, of whom three were still symptomatic. Other patients questioned said that it was difficult to wear knee pads at work as they got in the way. Atkins \& Marks $^{8}$ were able to show that kneg pads were not entirely successful in treatment of 'beat knee' in miners and the same appears to be true of th 8 patients reported here. Simple hygiene is probabl important in preventing the disease in workers who are constantly on their knees, ${ }^{6,8}$ and cleaning of prepatellas injuries may help to prevent infective prepatellap. bursitis. Preventing symptoms after infection is more difficult, and not surprisingly $75 \%$ of those wit persistent symptoms (nine patients) were employed io jobs which involved kneeling. None of the patients developed recurrent infection which suggests thas infection does not lower resistance to a further infec 3 tion in the bursa or elsewhere.

\section{Acknowledgements}

My thanks to the consultant staff of the Accident Service, John Radcliffe Hospital, Oxford for allowing me to report om their patients, and to $\mathrm{Mr}$ G.R. Houghton, M.A., F.R.C.S ${ }_{\in}^{\mathrm{NU}}$ for his advice.
2. Ho, G., Tice, A.D. \& Kaplan, S.R. Septic bursitis in th区 prepatellar and olecranon bursae. Ann Int Med 1978, 89. 21-27. 
3. Ho, G. \& Tice, A.D. Comparison of nonseptic and septic bursitis. Arch Int Med 1979, 139: 1269-1272.

4. Ho, G. \& Su, E.Y. Antibiotic therapy of septic bursitis. Its implication in the treatment of septic arthritis. Arthritis Rheum 1981, 24: 905-911.

5. Thompson, G.R., Manshady, B.M. \& Weiss, J.J. Septic bursitis. JAMA 1978, 240: 2280-2281.

6. Sharrard, W.J.W. Aetiology and pathology of beat knee. Br J Industr Med 1963, 20: 24-31.

7. Sharrard, W.J.W. Pressure effects on the knee in kneeling miners. Ann R Coll Surg Engl 1965, 36: 309-324.
8. Atkins, J.B. \& Marks, J. The role of staphylococcal infection in beat disorders of miners. Br J Industr Med 1952, 9: 296-302.

9. Editorial. Septic bursitis. Lancet, 1978, ii: 1135.

10. Ger, R. The skin incision in the excision of the prepatellar bursa. J Dermatol Surg Oncol 1978, 4: 166167.

11. Quayle, J.B. \& Robinson, M.P. An operation for chronic prepatellar bursitis. J Bone Joint Surg (Br) 1978, 58: 504-506. 